\title{
Inhibiting autophagy promotes endoplasmic reticulum stress and the ROS-induced nod-like receptor 3-dependent proinflammatory response in HepG 2 cells
}

\author{
JIA-JING YIN*, GUANGYING XIE* , NING ZHANG and YANBO LI \\ Department of Endocrinology, First Affiliated Hospital of Harbin Medical University, Harbin, Heilongjiang 150001, P.R. China
}

Received August 9, 2015; Accepted August 9, 2016

DOI: $10.3892 / \mathrm{mmr} .2016 .5708$

\begin{abstract}
Inflammation and endoplasmic reticulum (ER) stress are key contributors to insulin resistance and metabolic disease, and interleukin (IL)-1 $\beta$ is involved in insulin resistance. The present study aimed to investigated the role of autophagy in LPS-induced ER stress and inflammation, which may provide evidence for controlling metabolic disease associated with inflammation. Lipopolysaccharide (LPS) induced the activation of ER stress and the nod-like receptor 3-dependent expression of IL-1 $\beta$ and caspase-1, as shown by western blotting, which contributed to HepG2 cell death. This also involved the generation of mitochondrial reactive oxygen species and the autophagy signaling response, which are derived from the ER stress pathway. The percentage of apoptotic cells was measured by flow cytometry with fluorescein isothiocyanate/propidium iodide staining. Reactive oxygen species formation was detected by flow cytometry using the peroxide sensitive fluorescent probe 2',7'-dichlorofluorescin diacetate. Autophagy activation was measured by western blotting and confirmed using transmission electron microscopy. Furthermore, inhibiting autophagy promoted ER stress and the proinflammatory response in
\end{abstract}

Correspondence to: Professor Yanbo Li, Department of Endocrinology, First Affiliated Hospital of Harbin Medical University, 23 You Zheng Street, Harbin, Heilongjiang 150001, P.R. China

E-mail: liyanbo65@163.com

${ }^{*}$ Contributed equally

Abbreviations: ATG, autophagy-related gene; CHOP, C/EPB homologous protein; DCFH-DA, 2',7'-dichlorofluorescin diacetate; ER, endoplasmic reticulum; FBS, fetal bovine serum; IL-1 $\beta$, interleukin-1 $\beta$; LC3, light chain 3; LDL, low density lipoprotein; LPS, lipopolysaccharide; NAC, N-acetyl-L-cysteine; NLRP3, nod-like receptor pyrin domain-containing protein 3 ; ROS, reactive oxygen species; T2D, type 2 diabetes; TLR-4, toll-like receptor-4; TNF- $\alpha$, tumor necrosis factor- $\alpha$; UPR, unfolded response; 4-PBA, 4-phenyl butyrate; 3-MA, 3-methyladenine

Key words: autophagy, reactive oxygen species, endoplasmic reticulum stress, inflammation, apoptosis addition to cell death. These findings provide insights into the protective role of autophagy in LPS-induced cell death and ER stress, and further identified the association of autophagy, ER stress and inflammation in HepG2 cells.

\section{Introduction}

The hallmark of type 2 diabetes (T2D) is associated with low-grade systemic inflammation, characterized by upregulated cytokine production and the activation of inflammatory signaling pathways (1). Interleukin (IL)-1 $\beta$ is one of the major inflammatory cytokines involved in T2D. IL-1 $\beta$ is a risk factor for T2D by inducing insulin resistance in insulin-sensitive cells $(2,3)$. Hepatic inflammation is a complex process and originates in response to a variety of stresses (4), which are associated with systemic metabolic conditions, including non-alcoholic streatohepatitis, obesity, diabetes and metabolic syndrome (5).

Lipopolysaccharide (LPS) is known to be an endogenous danger mediator of the host inflammatory response to infection. It has been reported that LPS promotes the inflammatory responses by directly engaging Toll-like receptor-4 (TLR-4) and inducing the nuclear translocation of nuclear factor (NF)- $\mathrm{B}(6)$. In addition to TLRs, members of the nucleotide-binding oligomerization domain (NOD)-like receptor (NLR) family are important in inflammation and metabolism (7). NLR pyrin domain-containing 3 (NLRP3) inflammasome is one molecule of the NLR family, along with apoptotic speck protein containing a caspase recruitment domain and pro-caspase-1; these form molecular platforms termed inflammasomes, which mediate caspase- 1 activation, followed by the cleavage and release of the proinflammatory cytokines, interleukin (IL)-1 $\beta$ and IL-18 $(8,9)$. It has been demonstrated that a reduction in the adipose tissue expression of NLRP3 is coupled with decreased inflammation and improved insulin-sensitivity in obese patients with T2D (10). The ablation of NLRP3 prevents the obesity-induced inflammsome activation in fat depots and the liver, and enhances insulin-signaling (10). However, the mechanism underlying how the NLRP3-dependent inflammatory effects are generated and their role in LPS-induced HepG2 apoptosis remain to be fully elucidated.

To avoid biological and chemical inflammatory stimuli, cells rely on robust adaptive responses for the maintenance of cellular and histological homeostasis. Key processes are the endoplasmic reticulum (ER) stress response, autophagy 
and redox stress (11). ER stress signaling, referred to as the unfolded response (UPR), is triggered by three downstream proteins: PKR-like eukaryotic initiation factor 2 kinase, activating transcription factor 6 and inositol requiring $1 \alpha$. Mild ER stress activates the UPR responsible for the recovery of homeostasis (12). By contrast, protracted and excessive signaling via ER stress sensors is associated with the initiation of a mitochondrial pathway of apoptosis, which involves the transcription factor, C/EPB homologous protein (CHOP) (13). A causal link between the ER stress response and the apoptosis of hepatocytes has been established in a wide range of hepatic disorders. For example, ER stress-induced hepatocyte apoptosis in acute liver failure (14), increased ER stress during the development of non-alcoholic fatty liver disease and reduced ER stress decreased hepatic cell death, together with recovery of autophagic flux (15).

Autophagy is an evolutionarily conserved, lysosome-dependent system in eukaryotes, which regulates the turnover of cellular proteins and organelles. In hepatocytes, autophagy contributes to the removal of damaged mitochondria (16) and controls intracellular lipid metabolism (17). The selective autophagic degradation of mitochondria is known as mitophagy (18). Autophagy has been shown to control a variety of functions, including the control of innate and adaptive immune responses by regulating cytokine production $(19,20)$ and combatting persistent ER stress (21). It has been demonstrated that the downregulation of hepatic autophagy in obesity results in increased ER stress and insulin resistance (22). Macrophages derived from autophagy-related gene (ATG)16L1-deficient mice have been shown to produce higher levels of IL-1 $\beta$ (23), whereas mice with a conditional deletion of ATG7 in the intestinal epithelium show enhanced expression of IL-1 $\beta$ (24). The role of autophagy in hepatocyte inflammation remains to be fully elucidated, however, the NLRP3 inflammasome contributes to hepatocyte injury and inflammation (25). In the present study, the activation of ER stress and NLRP3-dependent inflammation were examined in LPS-induced HepG2 cells, and the role of autophagy in response to this stress was investigated in order to interpret the association between autophagy, ER stress and inflammation in hepatocytes.

\section{Materials and methods}

Reagents and antibodies. Fetal bovine serum (FBS) was purchased from (GE Healthcare Life Sciences, Coelbe, Germany). Dulbecco's modified Eagle's medium (DMEM) and $1 \%(\mathrm{v} / \mathrm{v})$ streptomycin/penicillin were purchased from Gibco; Thermo Fisher Scientific, Inc, (Waltham, MA, USA). LPS, 4-phenyl butyrate (4-PBA), 3-methyladenine (3-MA) and $N$-acetyl-L-cysteine (NAC) were purchased from Sigma-Aldrich; Thermo Fisher Scientific, Inc. Complete protease inhibitor mixture and immunoblot polyvinylidene difluorid (PVDF) membranes were purchased from Roche Diagnostics (Barcelona, Spain). RIPA lysis buffer and the BCA protein assay kit were purchased from Beijing ComWin Biotech Co., Ltd. (Beijing, China). Western Chemiluminescent Horseradish Peroxidase (HRP) substrate was purchased from EMD Millipore (Billerica, MA, USA). Rabbit anti-microtubule-associated protein 1 light chain 3 (LC3; 1:1,000; cat. no. 2775), mouse anti-C/EBP homologous protein (CHOP; 1:1,000; cat. no. 2895), rabbit anti-caspase-1 (1:1,000; cat. no. 2225), rabbit anti-IL-1 $\beta$ (cat. no. 2022) and rabbit $\beta$-actin $(1: 1,000$; cat. no. 8475) were purchased from Cell Signaling Technology, Inc. (Beverly, MA, USA).

Cell culture and stimulation. HepG2 cells (ATCC, Manassas, VA, USA) were cultured in DMEM, supplemented with $10 \%$ FBS. The cells were maintained at $37^{\circ} \mathrm{C}$ in an atmosphere of $5 \% \mathrm{CO}_{2}$ and $100 \%$ humidity. The cells $\left(1 \times 10^{4}\right)$ were pretreated with 3-MA $(2.5 \mathrm{mM}), 4-\mathrm{PBA}(0.1 \mathrm{mg} / \mathrm{ml})$ or NAC $(5 \mathrm{mM})$, respectively, for $1 \mathrm{~h}$, followed by treatment with $0.1 \mathrm{mg} / \mathrm{ml} \mathrm{LPS}$ for $24 \mathrm{~h}$ at $37^{\circ} \mathrm{C}$.

Transmission electron microscopy (TEM). The HepG2 cells were fixed in phosphate buffer $(\mathrm{pH} 7.4)$ containing $2.5 \%$ glutaraldehyde and $2 \%$ paraformaldehyde at room temperature for $60 \mathrm{~min}$. The cells were post-fixed in $1 \% \mathrm{OsO}_{4}$ at room temperature for $60 \mathrm{~min}$, dehydrated through graded ethanol solutions and embedded in Quetol 812 (Nisshin EM Co., Ltd., Tokyo, Japan). The regions containing the cells were block-mounted and cut into $70 \mathrm{~nm}$ sections, which were stained with uranyl acetate (saturated aqueous solution) and lead citrate, and examined using TEM (H-7100; Hitachi, Ibaraki, Japan).

Western blot analysis. The cells were washed with phosphate-buffered saline (PBS) and lysed in lysis buffer comprising 0.5\% Triton X-100, $10 \mathrm{mM}$ HEPES ( $\mathrm{pH} 7.9$ ), $50 \mathrm{mM} \mathrm{NaCl}, 100 \mathrm{mM}$ EDTA and $0.5 \mathrm{M}$ sucrose, with $0.1 \%$ protease inhibitor cocktail (Roche Diagnostics). The lysates were then incubated on ice for $30 \mathrm{~min}$ and centrifuged at $8,000 \mathrm{x} \mathrm{g}$ for $10 \mathrm{~min}$ at $4^{\circ} \mathrm{C}$. The total protien was quantified using a Bio-Rad Protein Assay kit (Bio-Rad Laboratories, Inc., Hertfordshire, UK). Equal quantities of protein were subjected to sodium dodecyl sulfate-polyacrylamide gel electrophoresis (10-15\%) and then transferred onto PVDF membranes. The molecular weights were estimated by comparison with a pre-stained protein ladder. Non-specific binding was blocked using 5\% skim milk. The membranes were then incubated with specific primary antibodies, as noted above, overnight at $4^{\circ} \mathrm{C}$. The membranes were then washed with PBS-Tween-20 and incubated with HRP-conjugated secondary antibodies: Anti-rabbit IgG (1:5,000; cat. no. 7074; Cell Signaling Technology, Inc.). The protein bands were detected using Western Chemiluminescent HRP substrate (EMD Millipore). The immunoblots were quantified by densitometric analysis using ImageTool 3.0 software (Adobe Photoshop). The quantification of protein phosphorylation was normalized to the corresponding total protein expression, and the relative expression level of a specific protein was normalized to $\beta$-actin.

Flow cytometry for the analysis of apoptosis. The rates of apoptosis of the HepG2 cells were examined by flow cytometry using annexin V-fluorescein isothiocyanate (FITC)/propidium iodide (PI) staining. Briefly, the cells were treated, as described above, in each group for $24 \mathrm{~h}$. The cells were then harvested, washed and resuspended in PBS at a density of $1 \times 10^{6}$ cells. Apoptotic cell death was measured by double staining with annexin V-FITC and PI using an annexin V-FITC apoptosis detection kit (Beyotime Institute of Biotechnology, Shanghai, 
China) according to the manufacturer's protocol. Flow cytometric analysis was performed immediately following staining. Data acquisition and analysis were performed by flow cytometry using Cell Quest software.

ROS detection. The formation of intracellular ROS in the HepG2 cells was measured by flow cytometry using the peroxide-sensitive fluorescent probe: 2', 7'-dichlorofluorescin diacetate (DCFH-DA). The cells $\left(\sim 1 \times 10^{6}\right.$ cells $\left./ \mathrm{ml}\right)$ were cultured in 6-well plates and incubated for $24 \mathrm{~h}$ in eight groups. Subsequently, the cells were incubated with medium containing $10 \mu \mathrm{M}$ DCFH-DA for $20 \mathrm{~min}$ at $37^{\circ} \mathrm{C}$. Following incubation with the dye, the cells were harvested and washed three times with serum-free medium to remove the extracellular dye. The cells were then resuspended in ice-cold serum-free medium and placed on ice in the dark. The levels of intracellular peroxide were measured using a flow cytometer (BD FACSAria; BD Biosciences, Franklin Lakes, NJ, USA). The peak excitation wavelength for oxidized DCF-DA was $488 \mathrm{~nm}$ and the emission was $525 \mathrm{~nm}$.

Statistical analysis. The results are expressed as the mean \pm standard error of the mean. Comparisons of a single variable in more than two groups were analyzed using one-way analysis of variance followed by Tukey's multiple comparison test (GraphPad Prism; GraphPad Software Inc., La Jolla, CA, USA). Statistical analysis was performed using a paired and unpaired $t$-test between two groups, using SPSS 12.0 software (SPSS, Inc., Chicago, IL, USA). $\mathrm{P}<0.05$ was considered to indicate a statistically significant difference.

\section{Results}

LPS activates the ROS-dependent NLRP3 inflammasome. The production of mature caspase- 1 , which is required for the processing and production of IL-1 $\beta$ and IL-18, requires two signals. The first, for the transcription and translation of pro-IL-1 $\beta$ and pro-IL-18, can be achieved by a number of stimuli, including LPS (26). The second signal is required to activate the inflammasome to cause the autocatalytic cleavage of pro-caspase-1 to caspase-1. The present study examined whether LPS induced the production of IL-1 $\beta$ in HepG 2 cells with $24 \mathrm{~h}$ treatment (Fig. 1A). To biochemically assess inflammasome activation, the cleavage maturation of pro-IL-1 $\beta$ and pro-caspase- 1 were determined using immunoblot analysis. The cleavage of pro-IL-1 $\beta$ to IL-1 $\beta$ and pro-caspase- 1 to caspase- 1 were increased by exposure to LPS for $24 \mathrm{~h}$ (Fig. 1A).

ROS has an essential role in inflammasome activation (9). The present study examined ROS formation using DCF-DA as the detection reagent (27). Treatment of the HepG2 cells with LPS induced ROS activation (Fig. 1B). The generation of ROS in HepG2 cells was significantly enhanced by 2 -fold following treatment with LPS for $24 \mathrm{~h}$, which was inhibited by NAC, a ROS inhibitor (Fig. 1B). NAC also inhibited the LPS-induced IL-1 $\beta$ and caspase- 1 cleavage (Fig. 1A), indicating that the LPS-induced inflammasome activation was ROS-dependent.

LPS induces autophagy in HepG2 cells. Autophagosomes are recognized at the ultrastructural level as double-membrane vacuolar structures containing visible cytoplasmic contents, including glycogen, mitochondria and endoplasmic reticulum (28). TEM of the HepG2 cells treated with LPS showed several autophagsomes and autolysosomes (Fig. 2A). The cells were also pre-treated with the autophagy inhibitor, 3-MA, for $1 \mathrm{~h}$ prior to LPS treatment for $24 \mathrm{~h}$. 3-MA effectively inhibited the formation of autophagosomes by LPS (Fig. 2A).

A reliable marker of autophagy is the conversion of the ATG protein, LC3, from a soluble form (LC3-I) to a lipidized form (LC3-II), which stably associates with the membranes of autophagosomes (29). This conversion can be detected by either observing the formation of punctuate structures or by measuring the accumulation of LC3-II. In the present study, the expression of LC3-II increased in the LPS-stimulated hepatocytes with $24 \mathrm{~h}$ treatment (Fig. 2B). 3-MA significantly inhibited the conversion of LC3-I to LC3-II, which was consistent with the results of the TEM (Fig. 2B).

ER stress mediates LPS-induced apoptosis and autophagy in HepG 2 cells. Accumulating evidence suggests that ROS directly or indirectly affects ER homeostasis and protein folding (30). Therefore, the present study further analyzed CHOP, which is an ER stress marker and contributes to ER stress-induced apoptosis (31). An increase in the expression of CHOP was found following LPS treatment for $24 \mathrm{~h}$ (Fig. 3A), which was significantly inhibited by the ER stress inhibitor, 4-PBA (Fig. 3A). Furthermore, LPS-induced apoptosis was reversed partially by 4-PBA (Fig. 3B), suggesting that LPS-induced ER stress contributed to HepG2 cell apoptosis.

In mammalian cells, ER stress has been shown to facilitate the formation of autophagosomes, and the induction of autophagy enables the removal of toxic misfolded proteins (32). Therefore, the present study aimed to investigate whether ER stress is involved in LPS-induced autophagy. The levels of microtubule-associated protein LC3 were analyzed. As shown in Fig. 3A, LPS significantly induced the levels of LC3B-II, and this effect was abrogated by pretreatment of the cells with 4-PBA (Fig. 3A).

Inhibiting autophagy increases ER stress and inflammatory cytokines. Autophagy is a physiological process for the clearance of undesired injurious material, which protects the cells. However, persistent and excessive autophagy leads to cell death. To confirm the contribution of LPS in the induction of autophagy, the HepG2 cells were pretreated with 3 -MA for $1 \mathrm{~h}$, followed by treatment with LPS for $24 \mathrm{~h}$. The results showed that 3-MA caused a marked increase in apoptosis of the LPS-treated HepG2 cells (Fig. 4A), which indicated that the induction of autophagy by LPS was primarily protective. Furthermore, 3-MA increased LPS-induced ROS generation and the ROS-dependent expression of IL-1 $\beta$ (Fig. 1A), and increased the expression of the ER stress marker, CHOP (Fig. 3A). These results suggested that the protective role of autophagy in LPS-induced HepG2 cells may have contributed to ameliorating ER stress and the ROS-dependent expression of inflammatory cytokines.

\section{Discussion}

In the model of hepatic insulin resistance, its primary pathological consequence, involving the substantial loss of hepatocytes, originates from a complex crosstalk between 
A
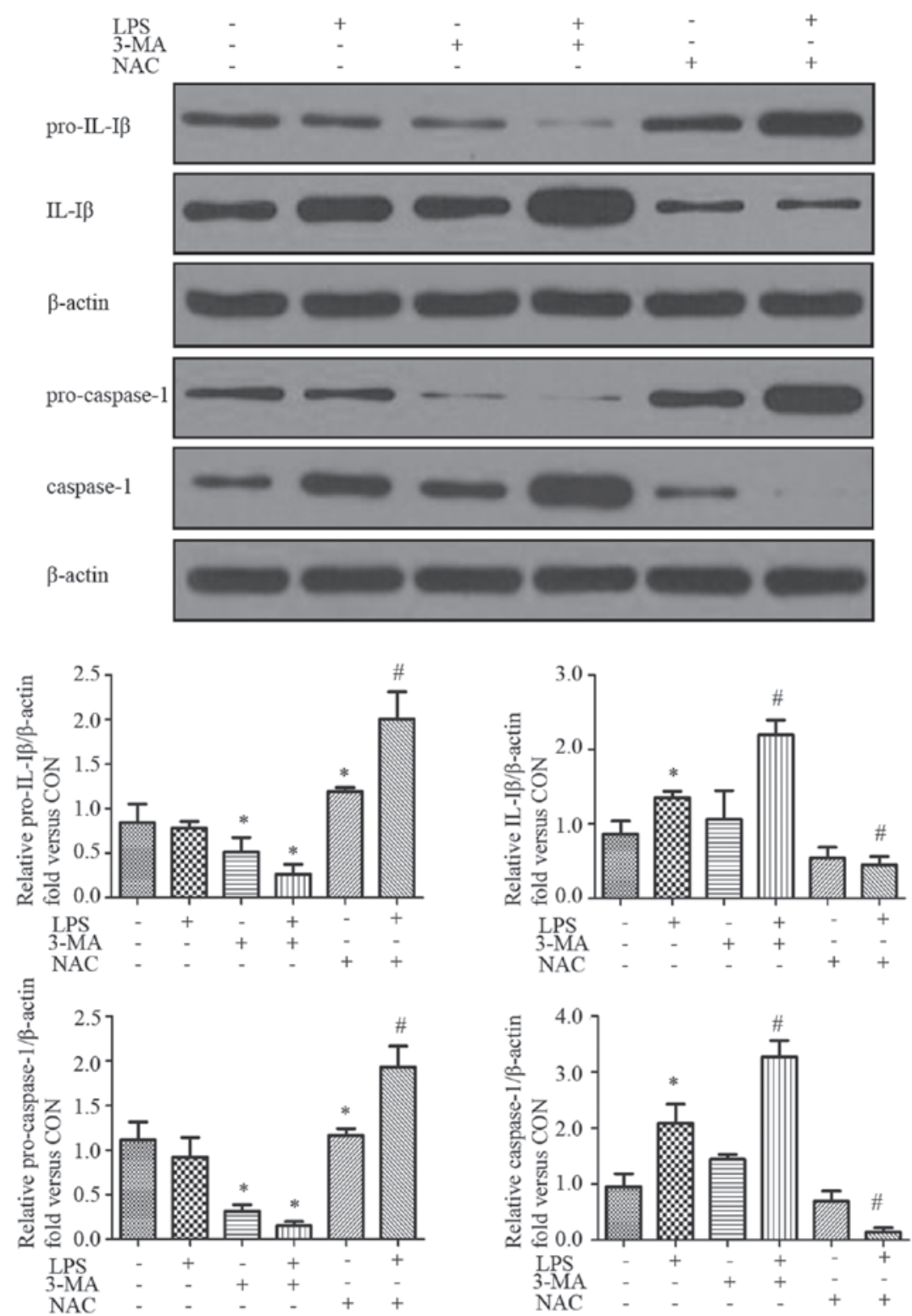

B

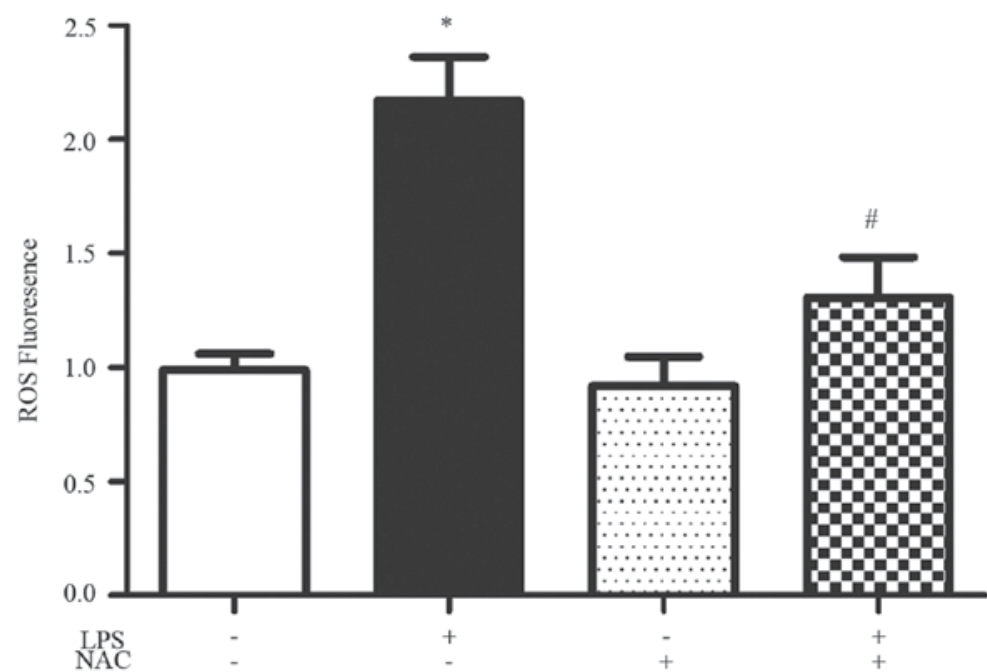

Figure 1. LPS activates ROS-dependent NLRP3 inflammasome. HepG2 cells were pretreated with or without 3-MA (2.5 mM) and/or NAC (5 mM) for $1 \mathrm{~h}$, followed with LPS $(0.1 \mathrm{mg} / \mathrm{ml})$ for $24 \mathrm{~h}$. (A) Western blot analysis was performed with the indicated antibodies. Proteins were quantified and normalized to $\beta$-actin. Representative blots and quantifications are shown. (B) ROS generation following treatment was evaluated using 2', 7'-dichlorofluorescin diacetate fluorescence (excitation, $488 \mathrm{~nm}$; emission, $525 \mathrm{~nm}$ ). Results are presented as the mean \pm standard error of the mean of 3-5 separate experiments. "P $<0.05$, vs. control group; ${ }^{*} \mathrm{P}<0.05$, vs. LPS-treated group. LPS, lipopolysaccharide; ROS, reactive oxygen species; NLRP3, NLR pyrin domain-containing protein 3; 3-MA, 3-methyladenine; NAC, $N$-acetyl-L-cysteine; IL, interleukin; CON, control (Dulbecco's modified Eagle's medium). 
A

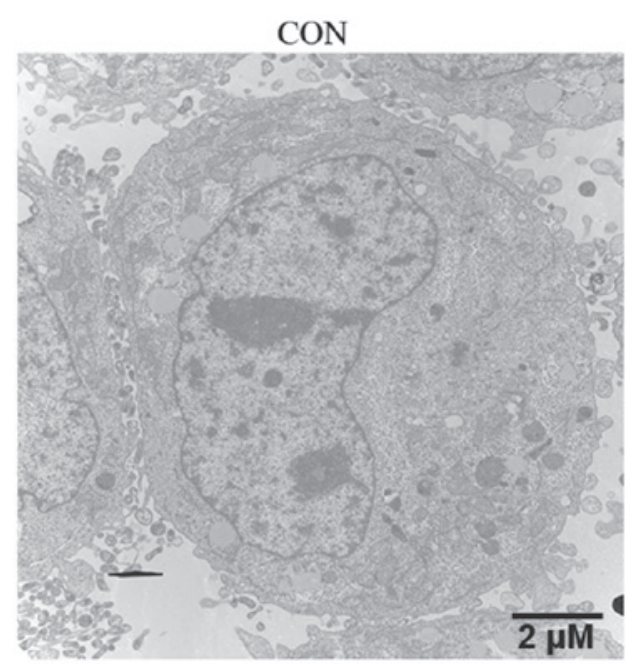

3-MA

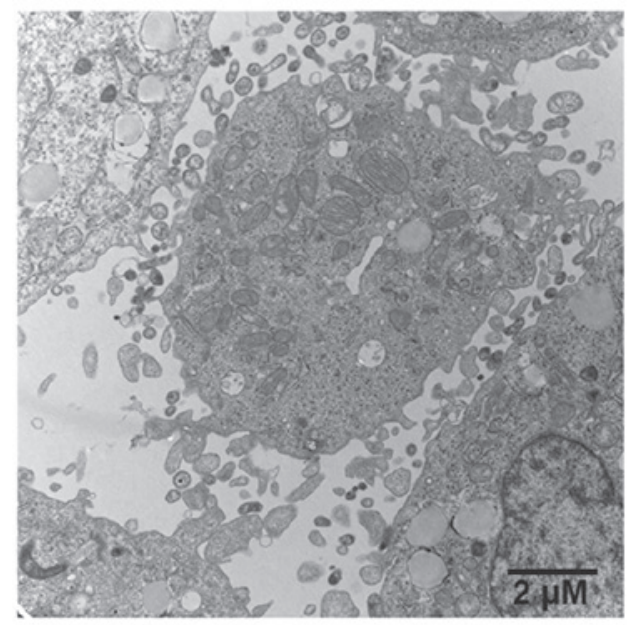

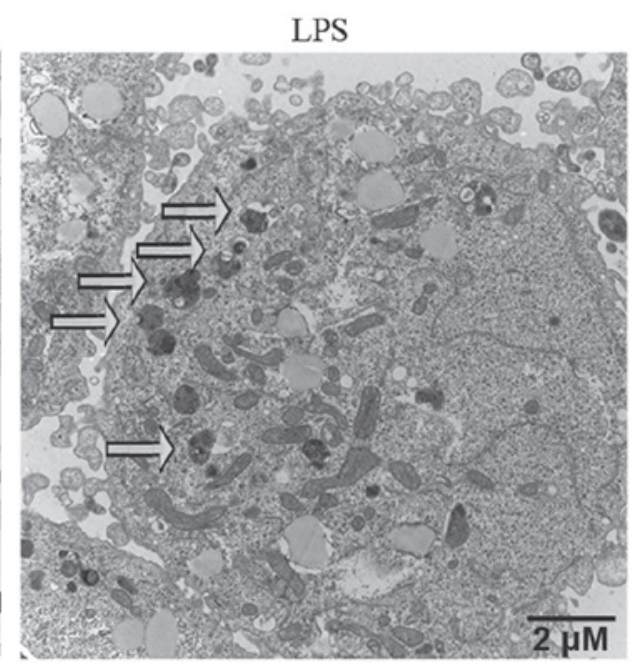

LPS+3-MA
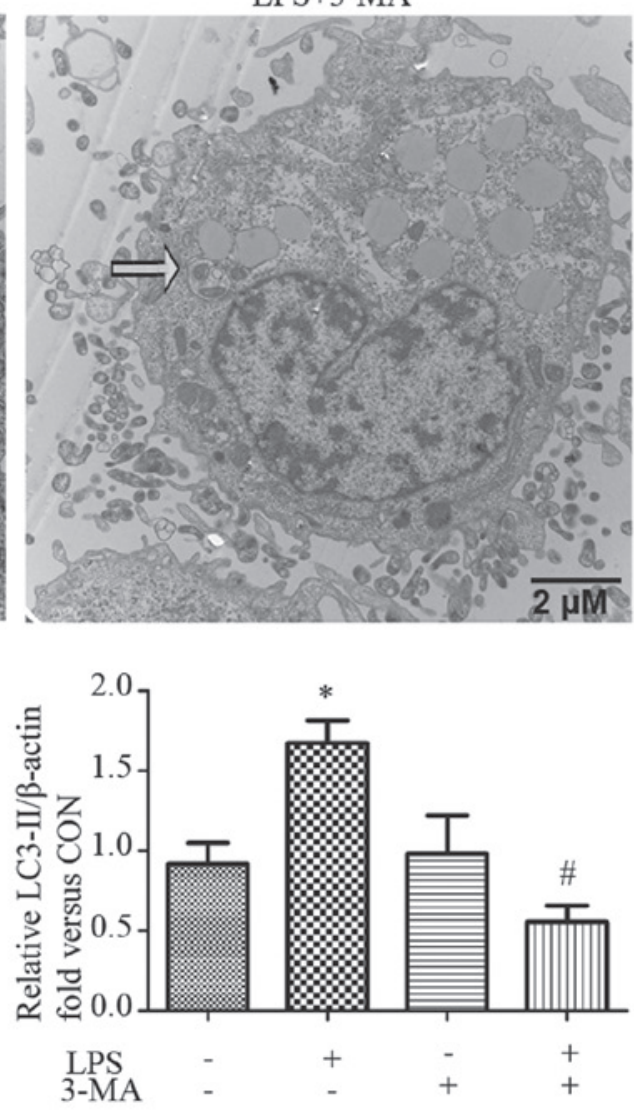

Figure 2. LPS induces autophagy in HepG2 cells. HepG2 cells were pretreated with or withour 3-MA (2.5 mM) for $1 \mathrm{~h}$, followed by treatment with LPS $(0.1 \mathrm{mg} / \mathrm{ml})$ for $24 \mathrm{~h}$. (A) Electron microscopy of treated HepG2 cells. Arrows represent autophagosomes and autolysosomes of double-membrane, single-membrane and multivesicular body-like vesicles. (B) Western blot analysis with the indicated antibodies. Proteins were quantified and normalized to $\beta$-actin. Representative blots and quantifications are shown. Results are presented as the mean \pm standard error of the mean of 3-5 separate experiments. ${ }^{\text {P }}<<0.05$. vs. control group; ${ }^{\#} \mathrm{P}<0.05$, vs. LPS-treated group. LPS, lipopolysaccharide; ROS, reactive oxygen species; 3-MA, 3-methyladenine; CON, control (Dulbecco's modified Eagle's medium).

ER stress, oxidative stress and inflammation during ischemia (11). Prominent signals leading to the substantial decline of hepatocytes, including tumor necrosis factor- $\alpha$ (TNF- $\alpha$ ), the overgeneration of ROS and the accumulation of misfolded proteins in the ER. These stimuli also mediate potent pro-inflammatory effects by promoting the activation of NF- $\kappa B$ or the NLRP3 inflammasome (11). Although several inflammatory cytokines have been indicated as pro-diabetic mediators, anti-inflammation-based therepeutic strategies targeting these cytokines in insulin resistance and T2D have been suboptimal $(33,34)$. The findings of the present study provided evidence for the activation of NLRP3-dependent inflammatory cytokines via ROS, induced by LPS in HepG2 cells, and that autophagy inhibition was responsible for the elevated levels of inflammation and ER stress induced by LPS, which contributed to HepG2 cell death. 
A
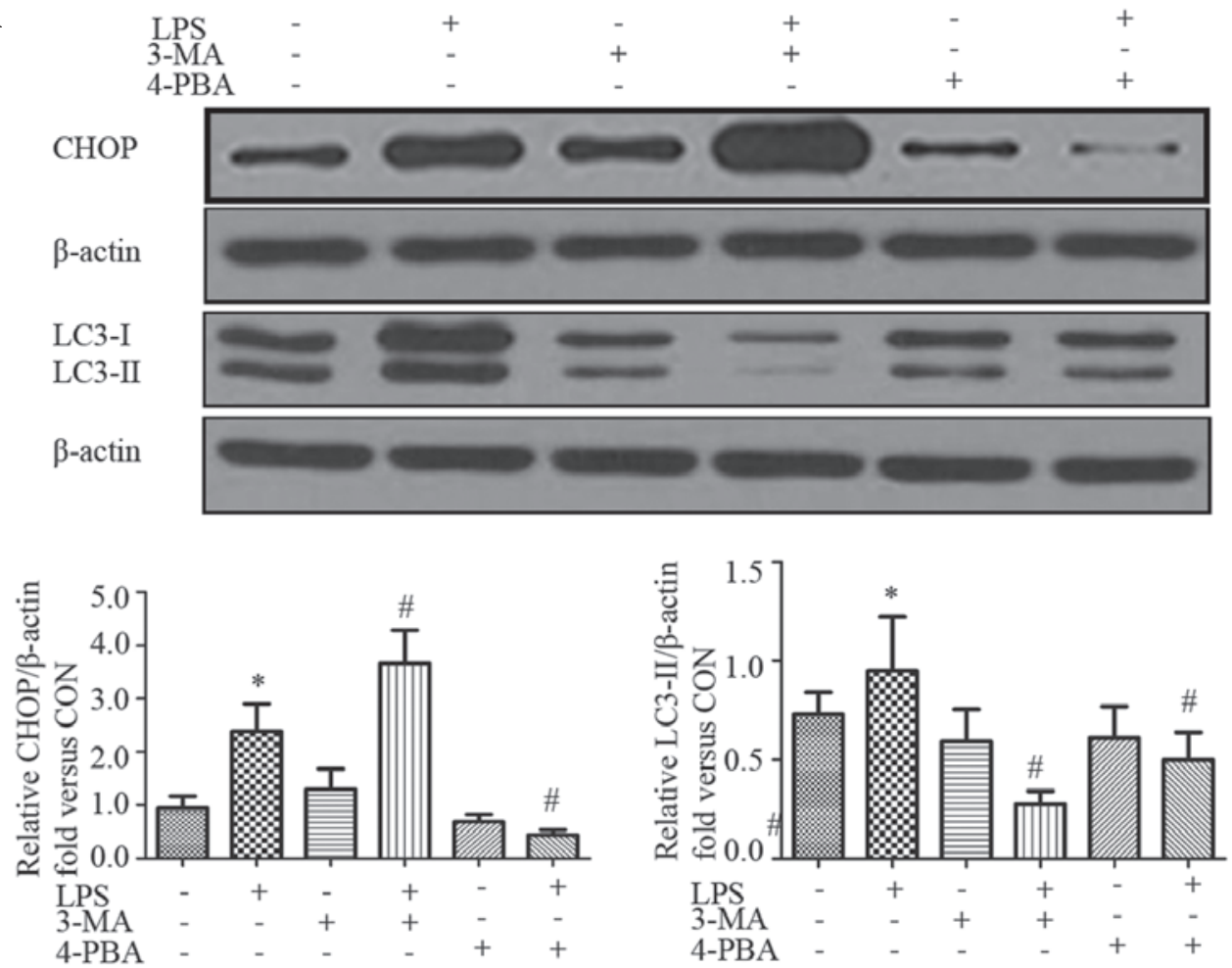

B

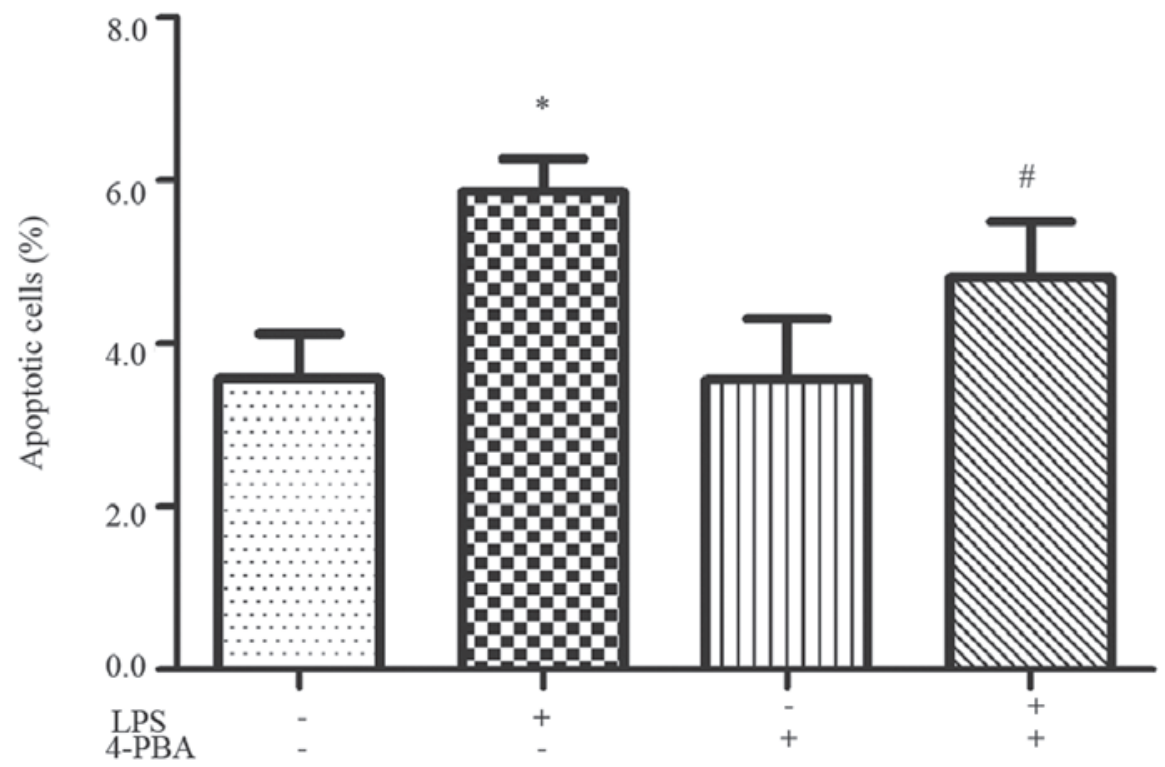

Figure 3. Endoplasmic retuculum stress mediates LPS-induced apoptosis and autophagy in HepG2 cells. HepG2 cells were pretreated with or without 3-MA $(2.5 \mathrm{mM})$ and/or 4-PBA $(0.1 \mathrm{mg} / \mathrm{ml})$ for $1 \mathrm{~h}$, followed with LPS $(0.1 \mathrm{mg} / \mathrm{ml})$ for $24 \mathrm{~h}$. (A) Western blot analysis was performed with the indicated antibodies. Proteins were quantified and normalized to $\beta$-actin. Representative blots and quantifications are shown. (B) Apoptotic cells were measured using Annexin-V/propidium iodide. Results are presented as the mean \pm standard error of the mean of 3-5 separate experiments. " $\mathrm{P}<0.05$, vs. control group; ${ }^{\#} \mathrm{P}<0.05$, vs. LPS-treated group. LPS, lipopolysaccharide; 3-MA, 3-methyladenine; 4-PBA, 4-phenyl butyrate; CHOP, C/EPB homologous protein; LC3. microtubule-associated protein light chain 3; CON, control (Dulbecco's modified Eagle's medium).

A wide variety of signals activate the NLRP3 inflammsome, and these include pathogen-associated molecular patterns (35) and host-derived molecules (9). The mechanisms by which these structurally distinct molecules trigger NLRP3 inflammasome activation remain to be fully elucidated. One of the suggested models states that NLRP3 is activated by a common pathway of ROS (36). The source of ROS remains to be fully elucidated, however, a previous study suggested the involvement of one or several of the seven known NADPH oxidases (36). In the present study, it was demonstrated that ROS was required for inflammasome activation in the HepG2 cells. During the progress of vascular inflammation, oxidized-LDL induces the production of ROS (37) and causes lysosomal damage (38), which are implicated in the mechanisms of NLRP3 inflammasome activation.

Products of the inflammasome are essential in the impairment of insulin signaling. Hepatocytes treated with IL-1 $\beta$ 
A

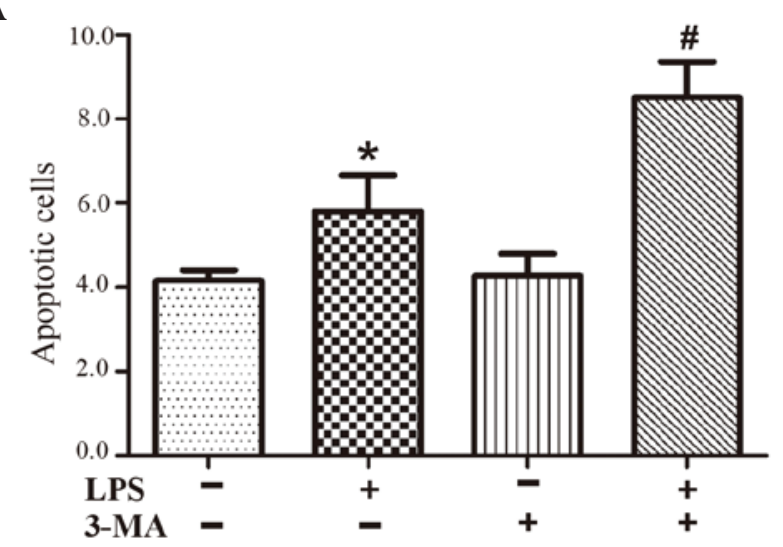

B

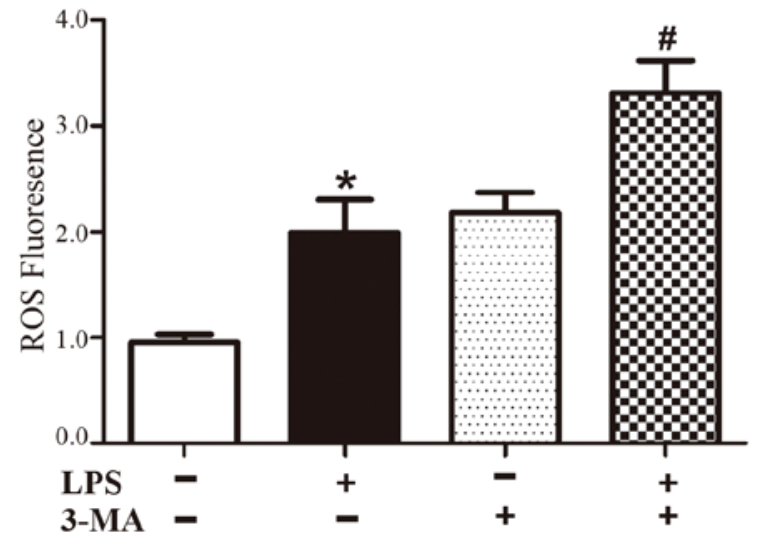

Figure 4. Inhibiting autophagy increases endoplasmic reticulum stress and the expression of inflammatory cytokines. The HepG2 cells were pretreated with or withour 3-MA $(2.5 \mathrm{mM})$ for $1 \mathrm{~h}$, followed by treatment with LPS $(0.1 \mathrm{mg} / \mathrm{ml})$ for $24 \mathrm{~h}$. (A) Percentages of apoptotic cells in the groups were measured using Annexin-V/propodium iodide. (B) ROS generation following treatment was evaluated using 2', 7'-dichlorofluorescin diacetate fluorescence (excitation, $488 \mathrm{~nm}$; emission, $525 \mathrm{~nm}$ ). The results are presented as the mean \pm standard error of the mean of 3-5 separate experiments. ${ }^{*} \mathrm{P}<0.05$, vs. control group (Dulbecco's modified Eagle's medium). ${ }^{\text {"}} \mathrm{P}<0.05$, vs. LPS-treated group. LPS, lipopolysaccharide; ROS, reactive oxygen species; 3-MA, 3-methyladenine.

or TNF- $\alpha$ show impaired Akt activation in response to insulin (26), consistent with clinical findings indicating that the IL-1 $\beta$ antagonist is promising for insulin resistance and T2D (39). In the present study, IL-1 $\beta$ was significantly increased following LPS treatment. The liver has a marked capacity to degrade LPS to which it is exposed almost continuously. The levels of LPS increase during hepatic injury and inflammation. Gandhi et al (40) demonstrated that LPS administration to rats caused mild liver injury and weight loss, however, all animals survived the endotoxin challenge. These observations suggested that the cell death-inducing and survival signals are stimulated by LPS in hepatocytes, with a predominance of the latter (28). The present study examined two responses, autophagy and ER stress, to LPS stimulation in HepG2 cells.

Persistent ER stress results in cell death and contributes to insulin resistance. The ER stress inhibitor, TUDCA, inhibits apoptosis by ameliorating ER stress through the modulation of intracellular calcium and thus attenuating liver cell death (41). By contrast, a previous study suggested that ER stress may be a source of the membranes during the formation of autophagic vesicles. In the present study, 4-PBA significant inhibited
LPS-induced LC3-II conversion, suggesting that the induced autophagy is, at least partly, dependent on ER stress. ER stress mediates polyglumaine-induced LC3 conversion, as essential step in formation of autophagy (42). ER stress negatively regulates the AKT/tuberous sclerosis complex/mammalian target of rapamycin pathway to enhance autophagy (43). In addition, ER stress leads to the release of calcium and subsequent activation of AMPK, which inhibits mROS, thereby promoting autophagy (44). In addition, ER stress-induced autophagy may have evolved as a mechanism used by cells to dispose of misfolded proteins, which cannot be degraded by ER-associated degradation, consequently assisting ER homeostasis (15). In the present study, the inhibition of autophagy by 3-MA significantly increased the expression of CHOP. Autophagy, as a cell survival mechanism, allows cells to remove damaged cytoplasmic proteins and organelles through lysosomal degradation and thus improving survival under metabolic stress (45). In the present study, the role of autophagy as a protective mechanism of cell survival was further confirmed by the autophagy inhibitor, 3-MA. The results demonstrated that 3-MA contributed to LPS-induced cell death, suggesting that autophagy had a protective role in this system

Substantial evidence indicates that autophagy is a potent suppressor of inflammation. Previously, Atg16L1 was demonstrated to control the production of inflammatory cytokines, including, TNF- $\alpha$, IL- 6 and IL- $1 \beta$, in response to LPS in macrophages (23). In the present study, inhbiting autophagy in HepG2 cells caused aberrant LPS-induced production of IL-1 $\beta$ and ROS. ROS may be accumulated in autophagy-deficient cells undergoing apoptosis and trigger the activation of caspase-1 following LPS stimulation. In myeloid cells and fatty acid metabolism in non-immune cells, inhibition of autophagy by pharmacological inhibitors or ATG deletion results in mitochondrial ROS generation, which activates NLRP3 $(46,47)$. Inhibiting autophagy with 3-MA in THP1 macrophages results in the accumulation of damaged mitochondria and increased concentrations of mitochondrial ROS (46). By preserving the functional pool of mitochondria, autophagy minimizes ROS generation, which inhibits the activation of intracellular pro-inflammatory factors, including the NFRP3 inflammasome and $\mathrm{NF}-\kappa \mathrm{B}$ (46). Autophagy acts as a tumor suppressor, likely by selectively removing damaged proteins and organelles, particularly damaged and senescent mitochondria, which are major cellular sources of ROS (48). However, reports have suggested that autophagy may promote the expression of pro-inflammatory cytokines and inflammation in certain biological contexts. For example, Hepatitis B virus-induced activation of $\mathrm{NF}-\kappa \mathrm{B}$ and release of inflammatory cytokines in hepatocytes is autophagy-mediated $(49,50)$. Further investigations on the mechanisms of autophagy during inflammation are required.

In conclusion, the data obtained in the present study showed that LPS induced the NLRP3-dependent proinflammatory response via ROS accumulation, as well as the activation of ER stress. Inhibiting autophagy increases ER stress and inflammatory cytokines. These findings provide evidence for the association of inflammation and ER stress with autophagy, and the protective role of autophagy in LPS-induced cell death and 
ER stress in HepG2 cells. However, it is possible that HepG2 cells vulnerable to death-inducing stimuli may be promoted by autophagy. Autophagy was shown to have a protective effect by ameliorating ER stress and NLRP3-dependent secretion of inflammatory cytokines, and further decreased cell death.

\section{Acknowledgements}

This study was supported by the National Natural Science Foundation of China (grant nos. 81370929 and 81400823), the Novo Nordisk China Diabetes Young Scientific Talent Research Funding (2013), the Research Fund for the Clinical Medicine of Chinese Medical Association (grant no. 13040670452) and the Science Foundation of the Education Department of Heilongjiang Province (grant no. 12531316).

\section{References}

1. Hotamisligil GS: Inflammation and metabolic disorders. Nature 444: 860-867, 2006.

2. Jager J, Grémeaux T, Cormont M, Le Marchand-Brustel Y and Tanti JF: Interleukin-1beta-induced insulin resistance in adipocytes through down-regulation of insulin receptor substrate-1 expression. Endocrinology 148: 241-251, 2007.

3. Lagathu C, Yvan-Charvet L, Bastard JP, Maachi M, Quignard-Boulangé A, Capeau J and Caron M: Long-term treatment with interleukin-1beta induces insulin resistance in murine and human adipocytes. Diabetologia 49: 2162-2173, 2006.

4. Kubes P and Mehal WZ: Sterile inflammation in the liver. Gastroenterology 143: 1158-1172, 2012.

5. Navab M, Gharavi N and Watson AD: Inflammation and metabolic disorders. Curr Opin Clin Nutr Metab Care 11: 459-464, 2008.

6. Amyot J, Semache M, Ferdaoussi M, Fontés G and Poitout V: Lipopolysaccharides impair insulin gene expression in isolated islets of Langerhans via toll-like receptor- 4 and $N F-\kappa B$ signalling. PloS one 7: e36200, 2012.

7. De Nardo D and Latz E: NLRP3 inflammasomes link inflammation and metabolic disease. Trends Immunol 32: 373-379, 2011.

8. Ting JP, Willingham SB and Bergstralh DT: NLRs at the intersection of cell death and immunity. Nat Rev Immunol 8: 372-379, 2008.

9. Schroder K and Tschopp J: The inflammasomes. Cell 140: $821-832,2010$

10. Vandanmagsar B, Youm YH, Ravussin A, Galgani JE, Stadler K, Mynatt RL, Ravussin E, Stephens JM and Dixit VD: The NLRP3 inflammasome instigates obesity-induced inflammation and insulin resistance. Nat Med 17: 179-188, 2011.

11. Brenner C, Galluzzi L, Kepp O and Kroemer G: Decoding cell death signals in liver inflammation. J Hepatol 59: 583-594, 2013.

12. Ogata M, Hino S, Saito A, Morikawa K, Kondo S, Kanemoto S, Murakami T, Taniguchi $\mathrm{M}$, Tanii I, Yoshinaga $\mathrm{K}$, et al: Autophagy is activated for cell survival after endoplasmic reticulum stress. Mol Cell Biol 26: 9220-9231, 2006.

13. Nishitoh H: CHOP is a multifunctional transcription factor in the ER stress response. J Biochem 151: 217-219, 2012.

14. Zhang L, Ren F, Zhang X, Wang X, Shi H, Zhou L, Zheng S, Chen Y, Chen D, Li L, Zhao C and Duan Z: Peroxisome proliferator-activated receptor alpha acts as a mediator of endoplasmic reticulum stress-induced hepatocyte apoptosis in acute liver failure. Dis Model Mech 9: 799-809, 2016.

15. González-Rodríguez A, Mayoral R, Agra N, Valdecantos MP, Pardo V, Miquilena-Colina ME, Vargas-Castrillón J, Lo Iacono O, Corazzari M, Fimia GM, et al: Impaired autophagic flux is associated with increased endoplasmic reticulum stress during the development of NAFLD. Cell Death Dis 5: e1179, 2014.

16. Green DR, Galluzzi L and Kroemer G: Mitochondria and the autophagy-inflammation-cell death axis in organismal aging. Science 333: 1109-1112, 2011.
17. Singh R, Kaushik S, Wang Y, Xiang Y, Novak I, Komatsu M, Tanaka K, Cuervo AM and Czaja MJ: Autophagy regulates lipid metabolism. Nature 458: 1131-1135, 2009.

18. Goldman SJ, Taylor R, Zhang Y and Jin S: Autophagy and the degradation of mitochondria. Mitochondrion 10: 309-315, 2010.

19. Klionsky DJ and Emr SD: Autophagy as a regulated pathway of cellular degradation. Science 290: 1717-1721, 2000.

20. Wang Y, Li YB, Yin JJ, Wang Y, Zhu LB, Xie GY and Pan SH: Autophagy regulates inflammation following oxidative injury in diabetes. Autophagy 9: 272-277, 2013.

21. Kuballa P, Nolte WM, Castoreno AB and Xavier RJ: Autophagy and the immune system. Annu Rev Immunol 30: 611-646, 2012.

22. Yang L, Li P, Fu S, Calay ES and Hotamisligil GS: Defective hepatic autophagy in obesity promotes ER stress and causes insulin resistance. Cell Metab 11: 467-478, 2010.

23. Saitoh T, Fujita N, Jang MH, Uematsu S, Yang BG, Satoh T, Omori H, Noda T, Yamamoto N, Komatsu M, et al: Loss of the autophagy protein Atg16L1 enhances endotoxin-induced IL-1beta production. Nature 456: 264-268, 2008.

24. Hotamisligil GS: Endoplasmic reticulum stress and the inflammatory basis of metabolic disease. Cell 140: 900-917, 2010.

25. Wree A, Eguchi A, McGeough MD, Pena CA, Johnson CD, Canbay A, Hoffman HM and Feldstein AE: NLRP3 inflammasome activation results in hepatocyte pyroptosis, liver inflammation and fibrosis in mice. Hepatology 59: 898-910, 2014.

26. Wen H, Gris D, Lei Y, Jha S, Zhang L, Huang MT, Brickey WJ and Ting JP: Fatty acid-induced NLRP3-ASC inflammasome activation interferes with insulin signaling. Nat Immunol 12: 408-415, 2011

27. LeBel CP, Ischiropoulos $\mathrm{H}$ and Bondy SC: Evaluation of the probe 2',7'-dichlorofluorescin as an indicator of reactive oxygen species formation and oxidative stress. Chem Res Toxicol 5: 227-231, 1992

28. Dangi A, Huang C, Tandon A, Stolz D, Wu T and Gandhi CR: Endotoxin-stimulated rat hepatic stellate cells induce autophagy in hepatocytes as a survival mechanism. J Cell Physiol 231: 94-105, 2016.

29. Czaja MJ, Ding WX, Donohue TM Jr, Friedman SL, Kim JS, Komatsu M, Lemasters JJ, Lemoine A, Lin JD, Ou JH, et al: Functions of autophagy in normal and diseased liver. Autophagy 9: 1131-1158, 2013.

30. Malhotra JD and Kaufman RJ: Endoplasmic reticulum stress and oxidative stress: A vicious cycle or a double-edged sword? Antioxid Redox Signal 9: 2277-2293, 2007.

31. Scull CM and Tabas I: Mechanisms of ER stress-induced apoptosis in atherosclerosis. Arterioscler Thromb Vasc Biol 31: 2792-2797, 2011

32. Ding WX, Ni HM, Gao W, Yoshimori T, Stolz DB, Ron D and Yin XM: Linking of autophagy to ubiquitin-proteasome system is important for the regulation of endoplasmic reticulum stress and cell viability. Am J Pathol 171: 513-524, 2007.

33. Uysal KT, Wiesbrock SM, Marino MW and Hotamisligil GS: Protection from obesity-induced insulin resistance in mice lacking TNF-alpha function. Nature 389: 610-614, 1997.

34. Sabio G, Das M, Mora A, Zhang Z, Jun JY, Ko HJ, Barrett T, Kim JK and Davis RJ: A stress signaling pathway in adipose tissue regulates hepatic insulin resistance. Science 322: 1539-1543, 2008.

35. Franchi L, Muñoz-Planillo R, Reimer T, Eigenbrod $\mathrm{T}$ and Núñez G: Inflammasomes as microbial sensors. Eur J Immunol 40: 611-615, 2010.

36. Dostert C, Pétrilli V, Van Bruggen R, Steele C, Mossman BT and Tschopp J: Innate immune activation through Nalp3 inflammasome sensing of asbestos and silica. Science 320: 674-677, 2008.

37. Napoli C, de Nigris F and Palinski W: Multiple role of reactive oxygen species in the arterial wall. J Cell Biochem 82: 674-682, 2001.

38. Yuan XM, Li W, Olsson AG and Brunk UT: The toxicity to macrophages of oxidized low-density lipoprotein is mediated through lysosomal damage. Atherosclerosis 133: 153-161, 1997.

39. Larsen CM, Faulenbach M, Vaag A, Vølund A, Ehses JA, Seifert B, Mandrup-Poulsen $T$ and Donath MY: Interleukin-1-receptor antagonist in type 2 diabetes mellitus. N Engl J Med 356: 1517-1526, 2007.

40. Gandhi CR, Kuddus RH, Nemoto EM and Murase N: Endotoxin treatment causes an upregulation of the endothelin system in the liver: Amelioration of increased portal resistance by endothelin receptor antagonism. J Gastroenterol Hepatol 16: 61-69, 2001. 
41. Xie Q, Khaoustov VI, Chung CC, Sohn J, Krishnan B Lewis DE and Yoffe B: Effect of tauroursodeoxycholic acid on endoplasmic reticulum stress-induced caspase-12 activation. Hepatology 36: 592-601, 2002.

42. Kouroku Y, Fujita E, Tanida I, Ueno T, Isoai A, Kumagai H, Ogawa S, Kaufman RJ, Kominami E and Momoi T: ER stress (PERK/eIF2alpha phosphorylation) mediates the polyglutamine-induced LC3 conversion, an essential step for autophagy formation. Cell Death Differ 14: 230-239, 2007.

43. Qin L, Wang Z, Tao L and Wang Y: ER stress negatively regulates AKT/TSC/mTOR pathway to enhance autophagy. Autophagy 6: 239-247, 2010.

44. Høyer-Hansen M and Jäättelä M: Connecting endoplasmic reticulum stress to autophagy by unfolded protein response and calcium. Cell Death Differ 14: 1576-1582, 2007.

45. Corcelle EA, Puustinen P and Jäättelä $M$ : Apoptosis and autophagy: Targeting autophagy signalling in cancer cells-'trick or treats? FEBS J 276: 6084-6096, 2009.

46. Zhou R, Yazdi AS, Menu P and Tschopp J: A role for mitochondria in NLRP3 inflammasome activation. Nature 469: 221-225, 2011
47. Nakahira K, Haspel JA, Rathinam VA, Lee SJ, Dolinay T, Lam HC, Englert JA, Rabinovitch M, Cernadas M, Kim HP, et al: Autophagy proteins regulate innate immune responses by inhibiting the release of mitochondrial DNA mediated by the NALP3 inflammasome. Nat Immunol 12: 222-230, 2011.

48. Degenhardt K, Mathew R, Beaudoin B, Bray K, Anderson D, Chen G, Mukherjee C, Shi Y, Gélinas C, Fan Y, et al: Autophagy promotes tumor cell survival and restricts necrosis, inflammation, and tumorigenesis. Cancer Cell 10: 51-64, 2006

49. Luo MX, Wong SH, Chan MT, Yu L, Yu SS, Wu F, Xiao Z, Wang X, Zhang L, Cheng AS, et al: Autophagy mediates $\mathrm{HBx}$-induced nuclear factor- $\kappa \mathrm{B}$ activation and release of IL-6, IL-8 and CXCL2 in hepatocytes. J Cell Physiol 230: 2382-2389, 2015.

50. Talero E, Alcaide A, Ávila-Román J, García-Mauriño S, Vendramini-Costa D and Motilva V: Expression patterns of sirtuin 1-AMPK-autophagy pathway in chronic colitis and inflammation-associated colon neoplasia in IL-10-deficient mice. Int Immunopharmacol 35: 248-256, 2016 